\title{
SPATIALLY RESOLVING THE VERY HIGH ENERGY EMISSION FROM MGRO J2019+37 WITH VERITAS
}

\author{
E. Aliu ${ }^{1}$, T. Aune ${ }^{2}$, B. Behera ${ }^{3}$, M. Beilicke ${ }^{4}$, W. Benbow ${ }^{5}$, K. Berger ${ }^{6}$, R. Bird ${ }^{7}$, A. Bouvier ${ }^{8}$, J. H. Buckley $^{4}$,
} V. Bugaev ${ }^{4}$, M. Cerruti $^{5}$, X. Chen $^{3,9}$, L. Ciupik ${ }^{10}$, M. P. Connolly ${ }^{11}$, W. Cui ${ }^{12}$, J. Dumm ${ }^{13}$, V. V. Dwarkadas ${ }^{14}$,

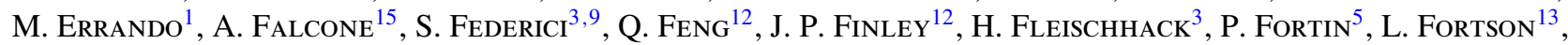
A. Furniss ${ }^{8}$, N. Galante ${ }^{5}$, G. H. Gillanders ${ }^{11}$, E. V. Gotthelf ${ }^{16}$, S. Griffin ${ }^{17}$, S. T. Griffiths ${ }^{18}$, J. Grube $^{10}$, G. Gyuk $^{10}$, D. Hanna ${ }^{17}$, J. Holder ${ }^{6}$, G. Hughes ${ }^{3}$, T. B. Humensky ${ }^{19}$, C. A. Johnson ${ }^{8}$, P. KaARet ${ }^{18}$, O. Kargaltsev ${ }^{20}$, M. Kertzman ${ }^{21}$, Y. Khassen ${ }^{7}$, D. Kiedi ${ }^{22}$, F. Krennrich ${ }^{23}$, M. J. LAng ${ }^{11}$, A. S Madhavan ${ }^{23}$, G. Maier ${ }^{3}$, S. McArthur $^{24}$, A. McCANn $^{25}$, J. Millis ${ }^{26,27}$, P. Moriarty ${ }^{27}$, R. Mukherjee ${ }^{1}$, D. Nieto ${ }^{19}$, A. O’Faoláin de Bhróithe ${ }^{7}$, R. A. Ong ${ }^{2}$, A. N. Otte ${ }^{28}$, D. Pandel ${ }^{29}$, N. Park ${ }^{24}$, M. Pohl ${ }^{3,9}$, A. PopKow ${ }^{2}$, H. Prokoph ${ }^{3}$, J. QuinN ${ }^{7}$, K. Ragan ${ }^{17}$, J. Rajotte ${ }^{17}$, L. C. ReYes ${ }^{30}$, P. T. ReYnolds ${ }^{31}$, G. T. Richards ${ }^{28}$, E. Roache ${ }^{5}$, M. Roberts ${ }^{32}$, G. H. Sembroski ${ }^{12}$, K. ShahinyAN ${ }^{13}$, A. W. SMith $^{22}$,

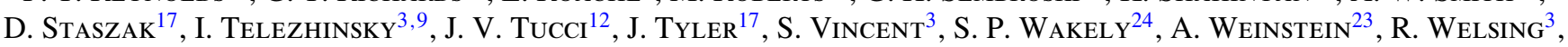
A. Wilhelm ${ }^{9}$, D. A. Williams ${ }^{8}$, AND B. Zitzer ${ }^{33}$

${ }^{1}$ Department of Physics and Astronomy, Barnard College, Columbia University, NY 10027, USA; ealiu @ astro.columbia.edu

${ }^{2}$ Department of Physics and Astronomy, University of California, Los Angeles, CA 90095, USA

${ }^{3}$ DESY, Platanenallee 6, D-15738 Zeuthen, Germany

${ }^{4}$ Department of Physics, Washington University, St. Louis, MO 63130, USA

${ }^{5}$ Fred Lawrence Whipple Observatory, Harvard-Smithsonian Center for Astrophysics, Amado, AZ 85645, USA

${ }^{6}$ Department of Physics and Astronomy and the Bartol Research Institute, University of Delaware, Newark, DE 19716, USA

${ }^{7}$ School of Physics, University College Dublin, Belfield, Dublin 4, Ireland

${ }^{8}$ Santa Cruz Institute for Particle Physics and Department of Physics, University of California, Santa Cruz, CA 95064, USA

${ }^{9}$ Institute of Physics and Astronomy, University of Potsdam, D-14476 Potsdam-Golm, Germany

${ }^{10}$ Astronomy Department, Adler Planetarium and Astronomy Museum, Chicago, IL 60605, USA

${ }^{11}$ School of Physics, National University of Ireland Galway, University Road, Galway, Ireland

${ }^{12}$ Department of Physics, Purdue University, West Lafayette, IN 47907, USA

${ }^{13}$ School of Physics and Astronomy, University of Minnesota, Minneapolis, MN 55455, USA

${ }^{14}$ Department of Astronomy and Astrophysics, University of Chicago, Chicago, IL 60637, USA

15 Department of Astronomy and Astrophysics, 525 Davey Lab, Pennsylvania State University, University Park, PA 16802, USA

${ }^{16}$ Columbia Astrophysics Laboratory, Columbia University, New York, NY 10027, USA

${ }^{17}$ Physics Department, McGill University, Montreal, QC H3A 2T8, Canada

${ }^{18}$ Department of Physics and Astronomy, University of Iowa, Van Allen Hall, Iowa City, IA 52242, USA

${ }^{19}$ Physics Department, Columbia University, New York, NY 10027, USA

${ }^{20}$ Department of Physics, The George Washington University, Washington, DC 20052, USA

${ }^{21}$ Department of Physics and Astronomy, DePauw University, Greencastle, IN 46135-0037, USA

22 Department of Physics and Astronomy, University of Utah, Salt Lake City, UT 84112, USA

${ }^{23}$ Department of Physics and Astronomy, Iowa State University, Ames, IA 50011, USA

${ }^{24}$ Enrico Fermi Institute, University of Chicago, Chicago, IL 60637, USA; nahee@ uchicago.edu

${ }^{25}$ Kavli Institute for Cosmological Physics, University of Chicago, Chicago, IL 60637, USA

${ }^{26}$ Department of Physics, Anderson University, 1100 East 5th Street, Anderson, IN 46012, USA

${ }^{27}$ Department of Life and Physical Sciences, Galway-Mayo Institute of Technology, Dublin Road, Galway, Ireland

${ }^{28}$ School of Physics and Center for Relativistic Astrophysics, Georgia Institute of Technology, 837 State Street NW, Atlanta, GA 30332-0430, USA

${ }^{29}$ Department of Physics, Grand Valley State University, Allendale, MI 49401, USA

${ }^{30}$ Physics Department, California Polytechnic State University, San Luis Obispo, CA 94307, USA

${ }^{31}$ Department of Applied Physics and Instrumentation, Cork Institute of Technology, Bishopstown, Cork, Ireland

32 Eureka Scientific Inc., 2452 Delmer Street, Suite 100, Oakland, CA 94602-3017, USA

${ }^{33}$ Argonne National Laboratory, 9700 S. Cass Avenue, Argonne, IL 60439, USA

Received 2013 December 3; accepted 2014 April 4; published 2014 May 23

\section{ABSTRACT}

We present very high energy (VHE) imaging of MGRO J2019+37 obtained with the VERITAS observatory. The bright extended $\left(\sim 2^{\circ}\right)$ unidentified Milagro source is located toward the rich star formation region Cygnus-X. MGRO J2019+37 is resolved into two VERITAS sources. The faint, point-like source VER J2016+371 overlaps CTB 87, a filled-center remnant (SNR) with no evidence of a supernova remnant shell at the present time. Its spectrum is well fit in the $0.65-10 \mathrm{TeV}$ energy range by a power-law model with photon index $2.3 \pm 0.4$. VER $\mathrm{J} 2019+378$ is a bright extended $\left(\sim 1^{\circ}\right)$ source that likely accounts for the bulk of the Milagro emission and is notably coincident with PSR J2021+3651 and the star formation region Sh 2-104. Its spectrum in the range 1-30 TeV is well fit with a power-law model of photon index $1.75 \pm 0.3$, among the hardest values measured in the VHE band, comparable to that observed near Vela-X. We explore the unusual spectrum and morphology in the radio and X-ray bands to constrain possible emission mechanisms for this source.

Key words: gamma rays: stars - pulsars: individual (PSR J2021+3651) - supernovae: individual (CTB 87)

Online-only material: color figures 


\section{INTRODUCTION}

High-mass star formation (and death) has long been associated with the acceleration of very high energy (VHE; $>100 \mathrm{GeV}$ ) particles (Ginzburg \& Syrovatskii 1964) and gamma-ray emission (Montmerle 1979; Kaaret \& Cottam 1996). There is clear evidence of VHE particle acceleration in the products of stellar death such as pulsars, supernova remnant (SNR) shells and pulsar wind nebulae (PWNe). Star-forming regions produce copious kinetic power in other forms, such as winds from Wolf-Rayet and OB stars. Regions with stellar winds, from single, binary, or collections of stars, have been suggested as possible VHE gamma-ray emitting sites, but as of today, observational evidence of this is still very scarce (LemoineGoumard et al. 2011).

The Cygnus- $X$ region is one of the richest known regions of star formation in the Galaxy. It is also close by (at only $1.4 \mathrm{kpc}$ ) and is, therefore, an excellent laboratory to study highenergy particle acceleration related to high-mass star formation and death. Because there are several spiral arms in the same direction, care must be exercised in relating any individual source to Cygnus-X. The Milagro sky survey identified several bright and extended VHE gamma-ray sources in the general direction of Cygnus (Abdo et al. 2007b). However, each Milagro source has multiple possible counterparts at lower energies, which complicates unambiguous associations.

MGRO J2019+37, with a measured flux of about $80 \%$ of the Crab Nebula flux at $20 \mathrm{TeV}$ (Abdo et al. 2007b), is the brightest Milagro source in the region. Since its discovery, the nature of MGRO J2019+37 has been the subject of studies and speculation, yet it remains unknown. Its bright inner region has an extent of about $1^{\circ}$ and overlaps with several SNRs, H II regions, Wolf-Rayet stars, high-energy (HE) gamma-ray $(>100 \mathrm{MeV})$ sources, and a hard X-ray transient. A tentative association with the young, energetic radio and gamma-ray pulsar PSR J2021+3651 and its nebula, SNR G75.1+0.2, has been suggested (Abdo et al. 2007b). There is extended X-ray and radio emission associated with the pulsar, but the size (less than $10^{\prime}$, Roberts et al. (2008)) is significantly smaller than the VHE source measured with Milagro. Paredes et al. (2009) suggested that this pulsar alone is not able to power the whole emission of MGRO J2019+37 because the time required for the electrons to diffuse and to fill a region of $1^{\circ}$ (at an uncertain distance of $2-10 \mathrm{kpc}$ ) is larger than their cooling time. The same authors performed deep radio and near-infrared surveys to find other potential counterparts, and proposed the massive star-forming region associated with the HiI region Sharpless 104 (Sh 2-104) as a possible contributor to the VHE emission through wind collisions or interactions of protostar jets with the surrounding medium (e.g., Torres et al. 2004). Particle acceleration in shocks driven by the winds from the Wolf-Rayet stars in the young cluster Ber 87 in the Cyg OB1 association has also been proposed as an origin of the VHE gamma-rays (Bednarek 2007).

Several VHE instruments have reported results on the region near MGRO J2019+37 at energies below $10 \mathrm{TeV}$. Relatively short observations with the imaging atmospheric Cherenkov telescopes (IACTs) MAGIC and VERITAS led to upper limits consistent with the Milagro source being extended and hard (Bartko et al. 2008; Kieda 2008). Bartoli et al. (2012) recently reported a non-detection based on data from the air-shower array ARGO-YBJ and concluded that the source could be variable. Only the Tibet Air Shower array has confirmed the detection of an extended VHE source from the same direction, with a statistical significance of 5.8 standard deviations $(5.8 \sigma)$ (Amenomori et al. 2008).

In this paper, we report on new and deeper observations of the region around MGRO J2019+37 made with VERITAS. The new observations provide much better angular resolution than Milagro and better sensitivity than any of the previous VHE measurements. These observations enable us to map the VHE emission in an attempt to better understand its physical origin. The instrument and observations are described in Section 2, while analysis and results can be found in Section 3 . A multi-wavelength analysis of the possible counterparts to this emission and a general discussion are presented in Section 4. A short summary and conclusions are drawn in Section 5.

\section{VHE OBSERVATIONS}

VERITAS is an array of four IACTs designed for observations of astrophysical objects in the energy range from $100 \mathrm{GeV}$ to above $10 \mathrm{TeV}$. The instrument angular resolution $(68 \%$ containment) reaches 0.08 per event and its sensitivity for a point-source is $1 \%$ of the steady Crab Nebula flux above $300 \mathrm{GeV}$ for a $5 \sigma$ detection within $25 \mathrm{hr}$ of observation at a $20^{\circ}$ zenith angle. A review of the detector is given by Holder et al. (2006, 2008).

The first pointed observations of the region around MGRO J2019+37 with VERITAS took place in 2006 November, during its commissioning phase, when the array had only two telescopes. The accumulated $10 \mathrm{hr}$ of exposure did not lead to any detection (Kieda 2008). The region was re-observed during the VERITAS survey of the Cygnus region from 2007 to 2008 using the full array (Weinstein et al. 2009). Analysis of the survey data with an effective on-source exposure of $7 \mathrm{hr}$ revealed a hint of a VHE gamma-ray signal within the large extension of the Milagro source.

A dedicated observation of the region took place between 2010 April and 2010 December, resulting in $70 \mathrm{hr}$ available for analysis after data quality selection. These observations were taken using the so-called wobble mode method, in which the source is offset by a small, angular distance from the center of the field of view (FOV), alternating between 20 minute runs in the four cardinal directions on the sky. This method ensures a simultaneous background estimate in each run. The size of the offset was decided based on the expected very large extent of the source and to have the maximum coverage to all objects in the field. The first $46 \mathrm{hr}$ of the observations used an offset of 0.7 around PSR J2021+3651, while $21 \mathrm{hr}$ of the observations used an offset of 0.6 around the hard X-ray transient IGR J20188+3657, located near the centroid of MGRO J2019+37. An additional 4 hr were also taken with offsets of 0.5 from the SNR CTB 87 to increase the exposure on the source. In this study, we only present data from these dedicated observations. The zenith angle of the selected data set ranges from $5^{\circ}$ to $35^{\circ}$.

\section{VHE GAMMA-RAY ANALYSIS AND RESULTS}

\subsection{Analysis}

The results presented here were generated using one of the standard VERITAS event reconstruction packages, similar to the scheme described in Acciari et al. (2008). All results were then verified by using a second independent software package described in Cogan (2008). Air shower images fully contained 


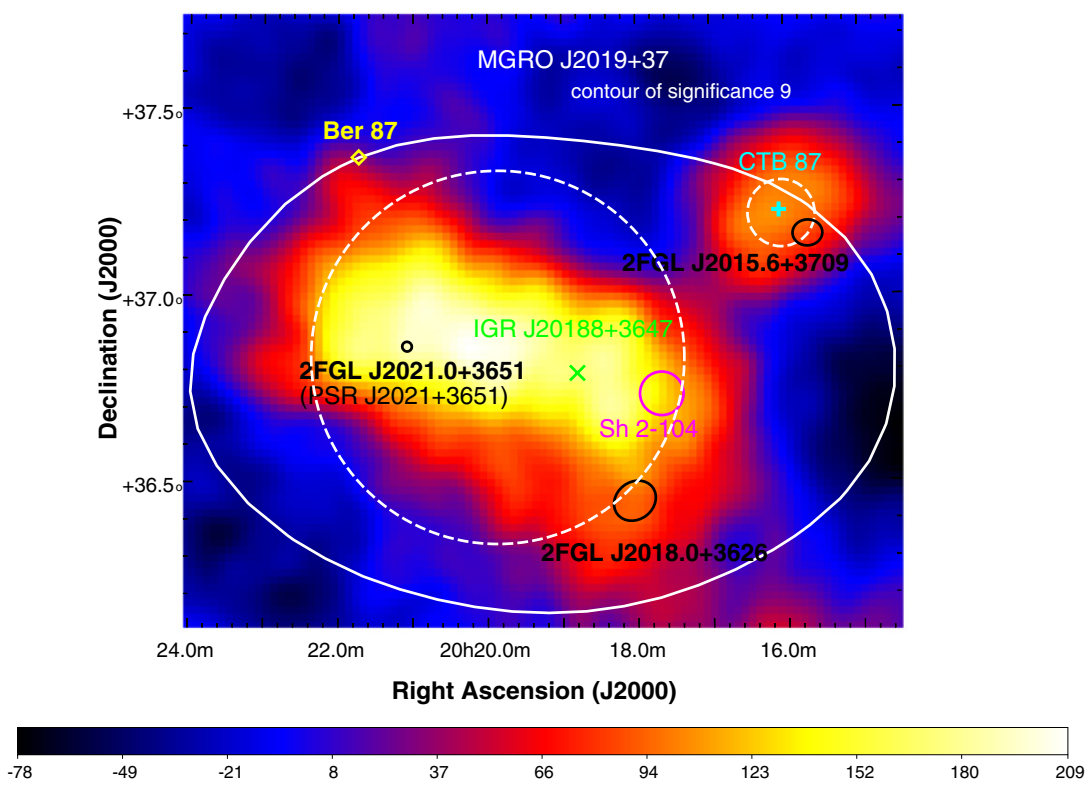

Figure 1. VHE gamma-ray excess map of the MGRO J2019+37 region as observed by VERITAS above $600 \mathrm{GeV}$. The color bar indicates the number of excess events within a search radius of 0.23 , which corresponds to the extended source search analysis described in the text. The shift between the red and blue color scale occurs at the $3 \sigma$ level. Regions used for spectral analysis of VER J2016+371 and J2019+368 are defined by white dashed circles. The locations of possible counterparts are marked using different colors. The contour of significance $9 \sigma$ of MGRO J2019+37 is overlaid in white.

(A color version of this figure is available in the online journal.)

in the individual telescope cameras are parameterized using the standard Hillas moment analysis (Hillas 1985). If the air shower image is not fully contained, a simple log-likelihood fitting of the image to a two-dimensional ellipse, which is generally accepted as a good representation of a gamma-ray shower (Hillas 1985), was applied in order to better estimate the shower parameters. The combination of both methods leads to more accurate reconstructions of off-axis events and more than a $30 \%$ increase of the effective area above $5 \mathrm{TeV}$, and thus to an improved sensitivity.

The spectral index of the gamma-ray source in the region, likely the counterpart of MGRO J2019+37, is expected to be hard $(\Gamma \sim 2)$ based on the previous result of Kieda (2008). However, the number of potential sources and their extensions are unknown. Therefore, we define two sets of selection criteria, for point sources and for extended sources, both optimized for weak emission with a hard spectral index. For the point source search, we use an integration radius of $\theta_{\text {int }}=0.089$, and for the extended source search, we use $\theta_{\text {int }}=0.23$. The optimized cuts require a hard cut on the image intensity, of 225 photoelectrons, yielding a mean energy threshold of $\sim 600 \mathrm{GeV}$.

For the background estimation of the sky image, the ring background method (Aharonian et al. 2005) was chosen with a background radius of 0.7 , to avoid possible gamma-ray contamination due to the spatial extent of the source. The reflected region background method (Aharonian et al. 2001) was used for the spectral analysis. Regions around stars with a magnitude less than 5.0 and the potential VHE sources PSR J2021+3651 and CTB 87 were excluded from the background estimation.

Trials have been conservatively estimated by tiling the area containing the Milagro source with 0.04 square search bins, which yields 2500 trials (1250 for each set of cuts) in the center region.
Table 1

Analysis Results

\begin{tabular}{lcrccc}
\hline \hline Source Name & $O^{\mathrm{a}}$ & \multicolumn{1}{c}{ Off $^{\mathrm{b}}$} & $\alpha^{\mathrm{c}}$ & Excess & ${\text { Significance }(\sigma)^{\mathrm{d}}}^{\mathrm{c}}$ \\
\hline VER J2016+371 & 126 & 317 & 0.181 & 69 & 7.0 \\
VER J2019+368 & 814 & 3656 & 0.160 & 228 & 8.2 \\
\hline
\end{tabular}

Notes.

a $O n$ is number of events in the source region.

b Off is number of events in the background region.

${ }^{\mathrm{c}} \alpha$ is defined by the ratio of the exposure of the source region to the exposure of background region.

d Significance shown here is before accounting for the trial factor.

\subsection{Results}

The VERITAS excess map of the MGRO J2019+37 region is shown in Figure 1, for the extended source search described previously. The map reveals at least two separate VHE emission regions where a single source had been reported by Milagro. Table 1 summarizes the details of the analysis result for each emission region.

One of the regions is located in the northwest of the FOV, where the gamma-ray excess is detected at a post-trials significance of $5.8 \sigma$ using the smaller search radius. This source is point-like to VERITAS, and its best fit position is found to be $\alpha_{J 2000}=20^{\mathrm{h}} 16^{\mathrm{m}} 2^{\mathrm{s}} \pm 3_{\text {stat }}^{s}, \delta_{J 2000}=37^{\circ} 11^{\prime} 52^{\prime \prime} \pm 40^{\prime \prime}{ }_{\text {stat }}$ and, hence, it is named VER J2016+371. This position is obtained by fitting a two-dimensional symmetric Gaussian $\left(\sigma_{x}=\sigma_{y}\right)$ function to the uncorrelated excess map. The systematic uncertainty in the measurement is well below $\sim 50^{\prime \prime}$. The position of this new VHE source is consistent with the peak of the radio SNR CTB 87, less than $1^{\prime}$ away (Kothes et al. 2003). The top panel in Figure 2 illustrates these results as well by showing the slices of the uncorrelated excess events of VER J2016+371. The directions of the slices are chosen along right ascension 
VER J2016+371 $37.1^{\circ} \leq \operatorname{Dec} \leq 37.3^{\circ}$

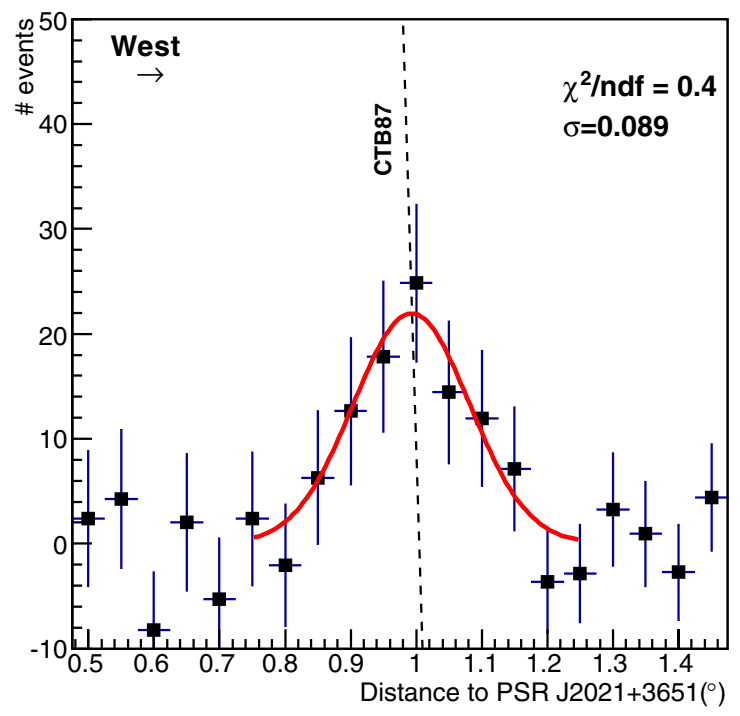

VER J2019+368 $36.75^{\circ} \leq$ Dec $\leq 36.95^{\circ}$

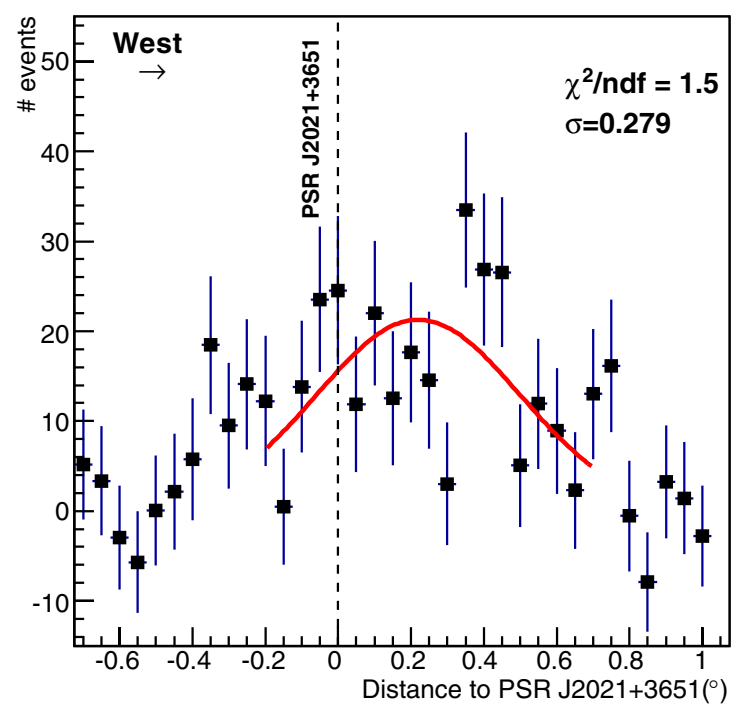

VER J2016+371 $303.9^{\circ} \leq \mathrm{RA} \leq 304.1$

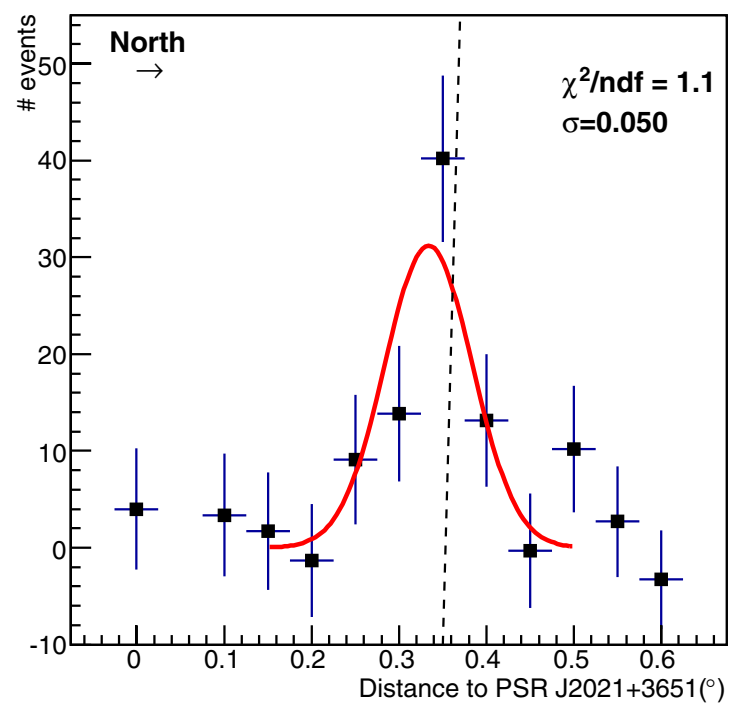

VER J2019+368 $304.25^{\circ} \leq \mathrm{RA} \leq 305.45^{\circ}$

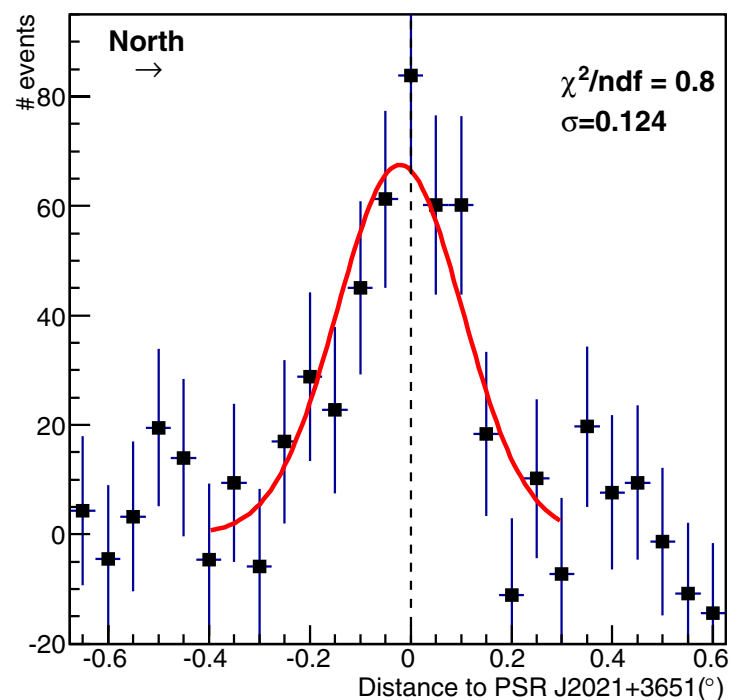

Figure 2. Slices in the uncorrelated excess maps of the new VHE sources. The width of the slice is indicated on each panel. The slices are centered on the best fit position of each VHE source. The direction of the slices follows right ascension. The top panels show the slices for VER J2016+371 and the peak position of the $1420 \mathrm{MHz}$ emission of CTB 87 (Kothes et al. 2003) is indicated with a dashed line. The bottom panels correspond to VER J2019+368 and the dashed line shows the position of the pulsar PSR J2021+3651.

(A color version of this figure is available in the online journal.)

(R.A.) and perpendicular to R.A., while the widths of the slices are chosen to be 0.2 . The energy spectrum of the VHE emission is obtained from a circular region of radius 0.09 centered on the nominal position of the SNR. This spectrum, shown in Figure 3, is well-described by a power-law (PL) model $\left(\chi^{2} /\right.$ dof $\left.=1.82 / 2\right)$ that extends from $650 \mathrm{GeV}$ up to $10 \mathrm{TeV}$ with a photon index of $\Gamma=2.3 \pm 0.3_{\text {stat }} \pm 0.2_{\text {sys }}$ and a differential flux at $1 \mathrm{TeV}$ of $\left(3.1 \pm 0.9_{\text {stat }} \pm 0.6_{\text {sys }}\right) \times 10^{-13} \mathrm{TeV}^{-1} \mathrm{~cm}^{-2} \mathrm{~s}^{-1}$. Using these best fit parameters, an integrated energy flux of $\left(8.2 \pm 3.4_{\text {stat }} \pm 2.9_{\text {sys }}\right) \times 10^{-13} \mathrm{erg} \mathrm{cm}^{-2} \mathrm{~s}^{-1}$ between 1 and $10 \mathrm{TeV}$ is obtained.

The other emission region lies in the center of the map in Figure 1. The result of the search with the larger radius shows emission in a region about one degree in extent and elongated along the R.A. axis, which is detected at a post-trial significance of $7.2 \sigma$. The centroid and extension of the emission were estimated by fitting a two-dimensional, asymmetric Gaussian $\left(\sigma_{x} \neq \sigma_{y}\right)$ function convolved with the VERITAS point-spread function to the uncorrelated excess event map. The centroid is located at $\alpha_{J 2000}=20^{\mathrm{h}} 19^{\mathrm{m}} 25^{\mathrm{s}} \pm 72_{\text {stat }}^{s}, \delta_{J 2000}=36^{\circ} 48^{\prime} 14^{\prime \prime} \pm$ $58^{\prime \prime}$ stat and, thus, labeled as VER J2019+368. From the fitting, the $1 \sigma$ angular extension of the emission was estimated to be $0.34 \pm 0.03_{\text {stat }}$ along the major axis, and $0.13 \pm 0.02_{\text {stat }}$ along the minor axis with the orientation angle of $71^{\circ}$ east of north. The extended emission region contains PSR J2021+3651 and its PWN, the HII region Sh 2-104 and the hard X-ray transient IGR J20188+3657, which are potential counterparts of the observed emission. To evaluate the possibility of various source contributions to VER J2019+368 we did a morphology test. We extracted the profile of the uncorrelated gamma-ray excess counts along the R.A. axis, see bottom panel of Figure 2, restricted to 0.2 in declination, and fitted several possible VHE morphologies, including a single extended source and a superposition of point sources. All fits resulted in similar 


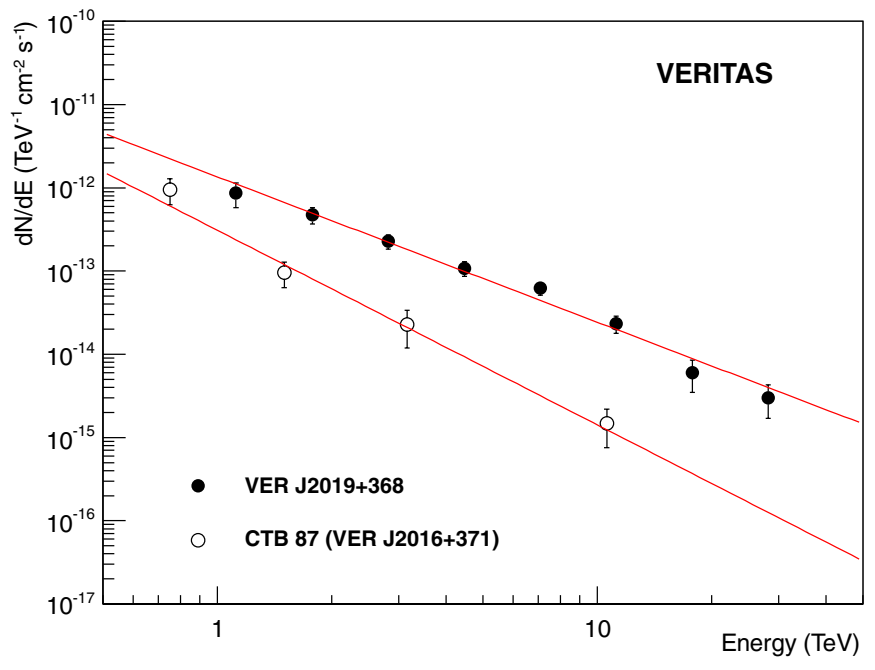

Figure 3. Differential energy spectrum of VER J2016+371/CTB 87 and VER J2019+368 as measured by VERITAS. The event excess in each bin have a statistical significance of at least $2 \sigma$.

(A color version of this figure is available in the online journal.)

reduced $\chi^{2}$ values, mainly due to limited statistics. Either a larger data set or more sensitive reconstruction techniques, or both, are necessary to determine the morphology better.

The energy spectrum for VER J2019+368 is estimated from a circular region of 0.5 radius centered on the best fit position. The resulting spectrum, shown in Figure 3, extends from 1 to $30 \mathrm{TeV}$ and is well fit by a PL model $\left(\chi^{2} /\right.$ dof $\left.=5.79 / 6\right)$ with a hard photon index of $\Gamma=1.75 \pm 0.08_{\text {stat }} \pm 0.2_{\text {sys }}$ and a differential flux at $5 \mathrm{TeV}$ of $\left(8.1 \pm 0.7_{\text {stat }} \pm 1.6_{\text {sys }}\right) \times$ $10^{-14} \mathrm{TeV}^{-1} \mathrm{~cm}^{-2} \mathrm{~s}^{-1}$. Assuming these parameters from the fit, the 1-10 TeV integrated energy flux is estimated to be $\left(6.7 \pm 0.5_{\text {stat }} \pm 1.2_{\text {sys }}\right) \times 10^{-12} \mathrm{erg} \mathrm{cm}^{-2} \mathrm{~s}^{-1}$. We also attempted to fit alternative, spectral models (such as a curved PL and cut-off PL model) but they did not provide better fits. The study of the energy dependent morphology of the emission in two separate energy bands, below $1 \mathrm{TeV}$, and above $1 \mathrm{TeV}$, supports the lack of any statistically significant spectral points below $1 \mathrm{TeV}$. The excess maps for each energy band show evidence for different centroid positions, see Figure 4. Above $1 \mathrm{TeV}$, a strong emission (at the level of $9 \sigma$ ) with a best fit location statistically compatible with that of VER J2019+368 is observed. Below $1 \mathrm{TeV}$, there are indications (at the level of $3 \sigma$ ) of emission offset by about 0.5 degrees in the direction of the unidentified gamma-ray source 2FGL J2018.0+3626.

\section{MULTIWAVELENGTH PROPERTIES, INTERPRETATION, AND DISCUSSION}

Both VHE-emitting regions coincide with non-thermal emission detected in radio, X-rays, and HE gamma-rays. In the following sections, we examine in detail the locations, morphologies, and spectral properties of these low energy counterparts in order to be able to establish the connection with the VHE emission and its origin.

\subsection{VER J2016+371, the SNR CTB 87, and their Surroundings}

In Figure 5 we present a false color image of the radio and X-ray emission in the region around VER J2016+371 obtained with the Giant Metrewave Radio Telescope (GMRT; Paredes et al. 2009) at $610 \mathrm{MHz}$ and Chandra between 2 and $10 \mathrm{keV}$,

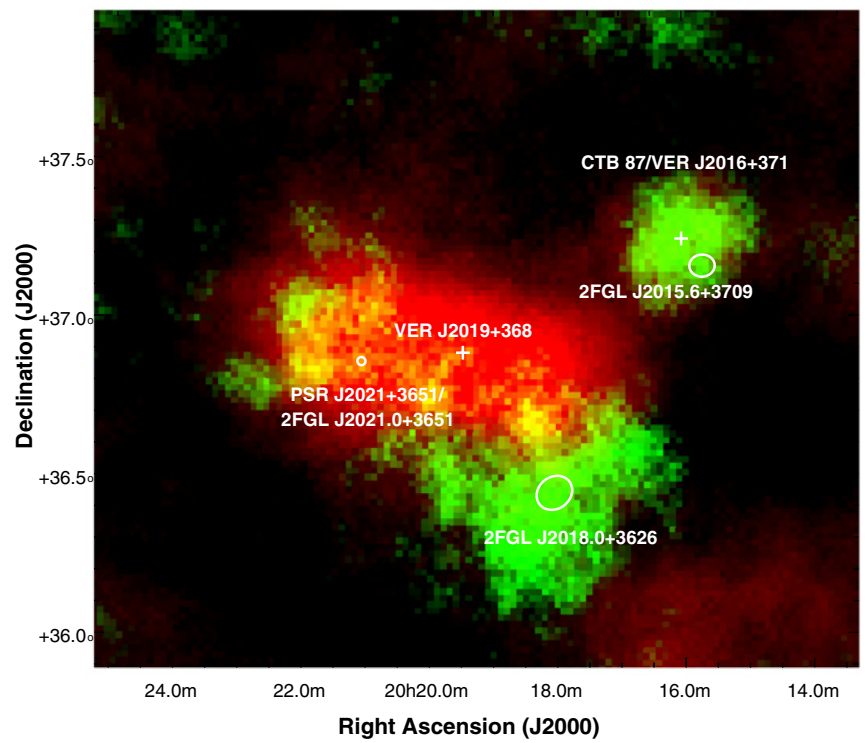

Figure 4. VHE gamma-ray excess maps of the MGRO J2019+37 region as observed with VERITAS in two different energy bands. The high energy band is above $1 \mathrm{TeV}$ (red) while the low energy band is between $600 \mathrm{GeV}$ and $1 \mathrm{TeV}$ (green). The number of excess events in the maps has been obtained using a search radius of 0.23 , which corresponds to the extended source search analysis described in the text. The change between the red and black in the color scale takes place at the $4 \sigma$ level, while between green and black is at the $2 \sigma$ level.

(A color version of this figure is available in the online journal.)

respectively. The VHE contours of VER J2016+371 are colocated with the bright and extended low-energy emission from the SNR CTB 87. At radio wavelengths, the strong polarization, flat spectral index, center-filled morphology, and lack of a continuum shell have been used to classify CTB 87 as a PWN (Weiler \& Shaver 1978; Wallace et al. 1997). The high angular resolution of the GMRT image $\left(\sim 30^{\prime \prime}\right)$ shows a faint circular structure in the southwestern portion of the nebula. Further studies at multiple wavelengths will be needed to determine if this structure is related to CTB 87 or perhaps a different source. The smoothed archival X-ray image reveals a centrallypeaked morphology which is offset toward the southeast of the radio peak and has a slightly smaller extent than the radio emission. The X-ray emission was recently studied in more detail by Matheson et al. (2013). The superb angular resolution of Chandra also allowed these authors to localize the pulsar candidate, CXOU J201609.2+371110, located within the compact PWN (to the southeast of the remnant center).

HE gamma-ray emission is also detected in the vicinity of VER J2016+371 with the Large Area Telescope on board the Fermi spacecraft (Fermi-LAT; Abdo et al. 2009b). The 95\% error ellipse of the unidentified HE gamma-ray source 2FGL J2015.6+3709 does not exclude a common origin between the two sources. However, based on the variability index of the Fermi-LAT source and its correlation with radio, Kara et al. (2012) associate the HE gamma-ray emission with the nearby blazar B2013+370, with unknown redshift, rather than with the CTB 87. On the other hand, no VHE gamma-ray emission from this extragalactic object is seen in the current data. Its location lies 6.7 away from the centroid of VER J2016+371, this being much larger than the $\sim 1.5$ uncertainty of the VHE measurement.

\subsubsection{A PWN scenario}

The morphology of the extended X-ray PWN (Matheson et al. 2013) suggests that it is affected by ram pressure due 


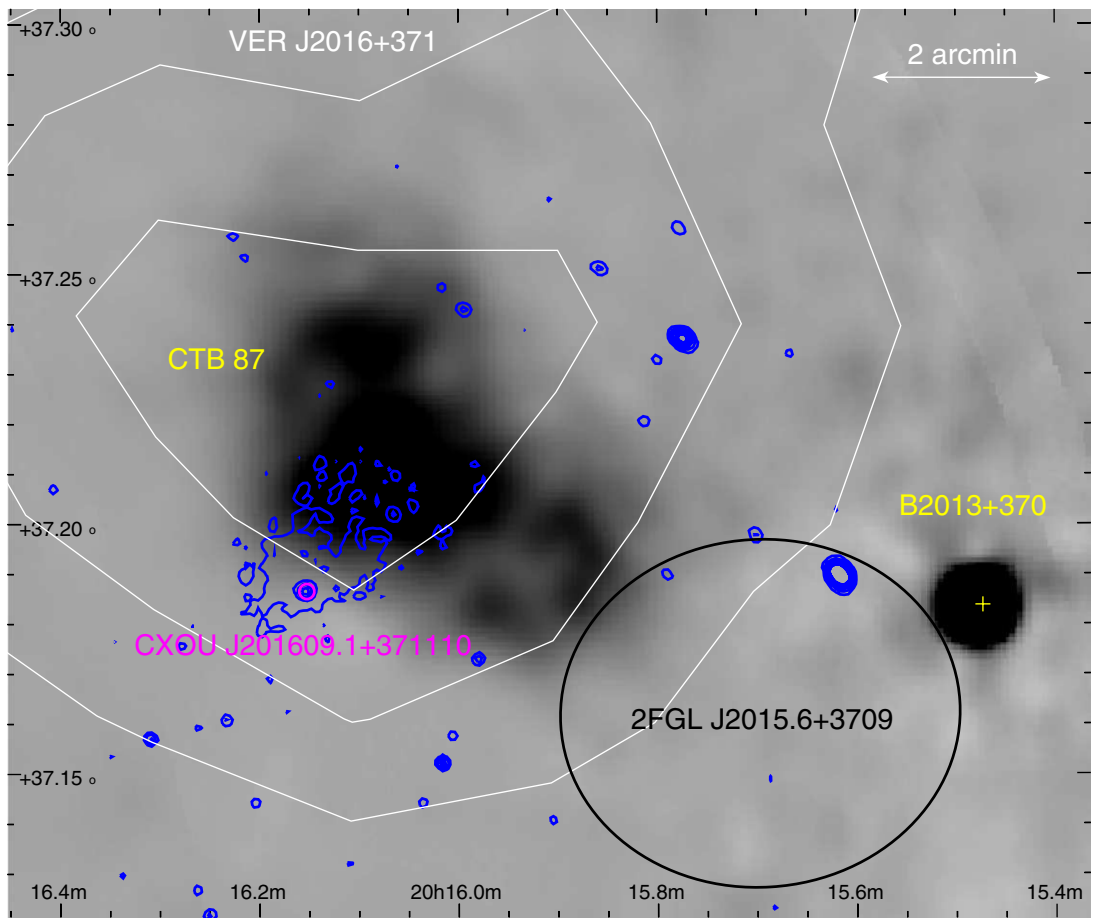

Figure 5. 610 MHz GMRT image of the CTB 87 region with X-ray and VHE gamma-ray contours in the equatorial coordinate system. The grayscale image is smoothed with a 2D Gaussian $\left(\sigma=30^{\prime \prime}\right)$. Blue contours indicate the X-ray morphology of the $0.3-7.5 \mathrm{keV}$ Chandra image with the position of the putative pulsar indicated in magenta. The VERITAS emission with an integration radius of 0.089 is shown as overlaid white contours of significance 3, 4, and $5 \sigma$. The blazar B2013+370 and the 95\% error ellipse of 2FGL J2015.6+3709, which are likely to be mutually associated, are also indicated.

(A color version of this figure is available in the online journal.)

to the proper motion of the pulsar which must then be moving southeast. Since the radio emission is not coincident with the compact $\mathrm{X}$-ray emission from the pulsar, the diffuse radio emission in the center of CTB 87 must be produced by an older generation of pulsar wind particles which have lost too much energy to emit X-rays via the synchrotron mechanism. A similar situation is seen for PSR B1706-44 in the SNR G343.1-2.3 (see Figure 2 of Romani et al. (2005)) which has been associated with the VHE source HESS J1708-443 (Abramowski et al. 2011). We note that the morphology of the compact X-ray PWN in CTB 87 (again, similar to PSR B1706-44 in G343.1-2.3) implies that the pulsars direction of motion is at a fairly large angle $\left(60^{\circ}-70^{\circ}\right)$ with respect to the compact PWN symmetry axis (which is also likely the pulsar spin axis).

If the pulsar was born at the apparent center of CTB 87 (i.e., $\sim 2^{\prime}$ away from the CXOU J201609.1+371110 position), the offset between the radio emission and the pulsar implies a transverse (projected onto the sky) velocity of $\simeq 70 d_{6.1} \tau_{50}^{-1} \mathrm{~km} \mathrm{~s}^{-1}$ where $d_{6.1}=d / 6.1 \mathrm{kpc}$ is the distance of the SNR, derived from H, I, and CO observations (Kothes et al. 2003), and $\tau_{50}=\tau / 50 \mathrm{kyr}$ an age scaled to an order of magnitude estimate based on the ages of known ram pressure confined $\mathrm{PWNe}$ resolved in X-ray at the distances $>2 \mathrm{kpc}$ (see pulsars marked by asterisks in Table 1 of Kargaltsev et al. (2013). Based on the fairly large X-ray luminosity, the pulsar could be younger than the reference age of $50 \mathrm{kyr}$ (see also Matheson et al. 2013). In this case the pulsar velocity would become supersonic compared to the sound velocity of heated ejecta $\left(\sim 100 \mathrm{~km} \mathrm{~s}^{-1}\right)$, or highly supersonic if the pulsar has completely escaped the unseen SNR into the warm ISM, where the sound velocity is $\sim 10 \mathrm{~km} \mathrm{~s}^{-1}$ (see, e.g., Gaensler et al. 2004).

Alternatively, the offset between the radio emission and the $\mathrm{X}$-ray nebula may largely be due to an asymmetric reverse shock that pushed the relic radio emitting PWN away from the pulsar's current position. In both scenarios, the PWN needs to be old enough for the reverse shock to have passed, making an age younger than 5-10 kyr unlikely.

In a commonly considered relic PWN scenario, where the $\mathrm{X}$-rays are attributed to synchrotron emission from pulsar wind and the VHE gamma-rays are interpreted as the cosmic microwave background photons up-scattered by pulsar wind electrons via the IC mechanism (see, e.g., Aharonian \& Atoyan 1995), the average magnetic field within the PWN can be estimated following arguments given in Aliu et al. (2013) for a PWN in the CTA 1 SNR. These estimates lead to a somewhat high $B_{\mathrm{PWN}} \sim 20-40 \mu \mathrm{G}$, if diffusion is neglected for the pulsar wind particles, and to a more reasonable $B_{\mathrm{PWN}} \sim 5 \mu \mathrm{G}$, if diffusion is the dominant transport mechanism (see Aliu et al. 2013 for details). The multiwavelength properties of VER J2016+371 are in line with those of other VHE PWNe (Kargaltsev et al. 2013); therefore, VER J2016+371 is another example of a PWN which is seen in both X-rays and VHE gamma-rays.

\subsection{VER J2019+368, the Main Contributor of MGRO J2019+37}

VER J2019+368 is an extended source that is about four times brighter than VER J2016+371 at $1 \mathrm{TeV}$. The centroid of the VER J2019+368 emission is separated from VER J2016+371 by $\sim 0.8$, and coincides well with the center region of MGRO J2019+37, as shown in Figure 1. The extension of VER $\mathrm{J} 2019+368$ is $\sim 0.35$ along the major axis, which is $50 \%$ smaller than the extension of 0.7 for MGRO J2019+37, as reported by Abdo et al. (2012). Figure 6 shows the spectral energy distribution of the MGRO J2019+37 region measured 


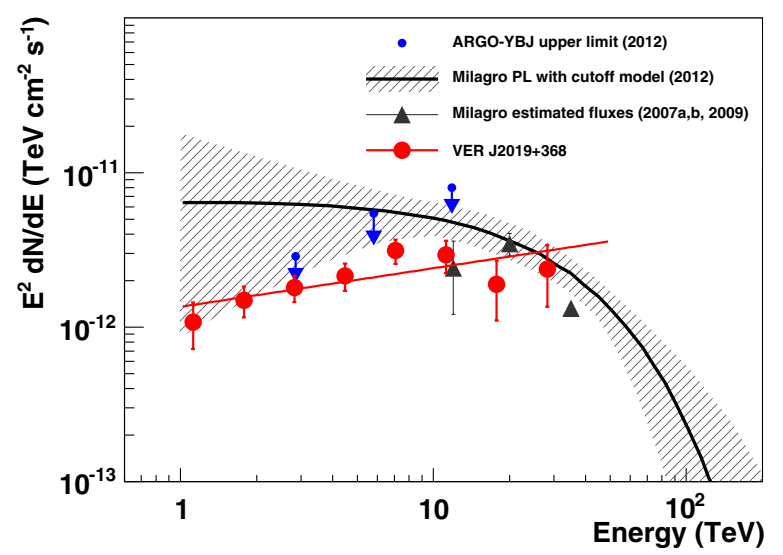

Figure 6. Spectral energy distribution of MGRO J2019+37/VER J2019+368 as measured by different instruments. VERITAS measures a spectrum from $1 \mathrm{TeV}$ to almost $30 \mathrm{TeV}$, shown in red, which is best fit with a power law with a hard spectral index. The Milagro flux points at 12, 20, and $35 \mathrm{TeV}$ are shown in black (Abdo et al. 2007a, 2007b, 2009a) and also in black is their best fit, a power law with a cutoff (Abdo et al. 2012). The shadowed area corresponds to the $1 \sigma$ band. ARGO-YBJ 90\% confidence level upper limits for MGRO J2019+37 are shown with blue arrows (Bartoli et al. 2012).

(A color version of this figure is available in the online journal.)

by different VHE gamma-ray instruments. Abdo et al. (2012) estimated the spectral index of MGRO J2019+37 to be $2.78 \pm$ 0.1 for a PL hypothesis and $2.0_{-0.1}^{+0.5}$ for a PL with a cut-off hypothesis. Their $F$-test favored the PL + cut-off model, for which the spectral index is in agreement with that measured by VERITAS for VER J2019+368, $1.75 \pm 0.38$. The spectrum of VER J2019+368 can be explained by a PL model up to $\sim 30 \mathrm{TeV}$. Even though the flux from CTB 87 is not included for VERITAS measurements (unlike Milagro's measurements including the emissions from a larger region including CTB 87), the spectra are consistent. As seen in Figure 6, the flux of VER J2019+368 is consistent with fluxes estimated by Milagro at 12, 20, and $35 \mathrm{TeV}$ and is also in agreement with the upper limit estimated by ARGO-YBJ. Based on the consistent flux levels and coincident location of the centroids between the two measurements, we expect VER J2019+368 to be the main contributor to the VHE emission from MGRO J2019+37.

Several surveys in radio, infrared, and X-ray wavelengths have studied the inner and brighter region of MGRO J2019+37 in an attempt to identify potential counterparts to the VHE emission. Since VER J2019+368 likely shares the counterparts of the inner MGRO J2019+37 and provides better localization, we are now able to reevaluate the possible sources and mechanisms generating the VHE emission. The potential counterparts suggested from the surveys can be found in Figure 7, marked over the radio continuum images of the region. These radio images are from the Dominion Radio Astrophysical Observatory within the Canadian Galactic Plane Survey (CGPS) project at $408 \mathrm{MHz}$ (Taylor et al. 2003) and the Green Bank Telescope at $4.85 \mathrm{GHz}$ (GB6). The VHE emission appears to follow a ridge of diffuse emission starting at the bright bubble H II region Sh 2-104 at the west end and roughly ending near the energetic gamma-ray pulsar PSR J2021+3651.

\subsubsection{WR 141}

This Wolf-Rayet $\mathrm{O}$ star colliding wind binary system is in the Cygnus arm at a distance of $\sim 1.3 \mathrm{kpc}$ (Van der Hucht 2001). The estimated terminal wind velocity and mass loss rates from the system suggest it is dumping energy into its surroundings at a rate of $\sim 2 \times 10^{37} \mathrm{erg} \mathrm{s}^{-1}$. While high-energy gamma-ray emission is theoretically expected to arise from such a system, Reimer et al. (2006) predicts a suppression of the highest energetic photons in the VHE band in the Klein-Nishina regime. In addition, there is nothing particularly remarkable about the X-ray, radio, and optical studies of WR 141 compared to similar systems that are not associated with bright VHE emission (Zhekov 2012; Montes et al. 2009; Marchenko et al. 1998), which makes this object a less likely counterpart for producing the observed VHE gamma-rays.

\subsubsection{PSR J2021+3651 and its $P W N$}

Located at $\sim 20^{\prime}$ eastward from the centroid of VER J2019+368, the young and energetic pulsar PSR J2021+3651 has a high spin-down luminosity $\left(\dot{E}=3.4 \times 10^{36} \mathrm{erg} \mathrm{s}^{-1}\right.$; Roberts et al. 2002). X-ray observations with Chandra revealed a compact X-ray nebula with a clearly-resolved torus and jet morphology surrounded by extended, diffuse emission (Hessels et al. 2004; Van Etten et al. 2008). According to the X-ray and VHE PWN population study by Kargaltsev \& Pavlov (2010), typically only pulsars with spin-down powers higher than $10^{35} \mathrm{erg} \mathrm{s}^{-1}$ are associated with prominent VHE PWNe. The high spin-down luminosity of PSR J2021+3651 makes a VHE PWN scenario for VER J2019+368 plausible.

To understand the overall picture, we performed a deep observation of the PSR J2021+3651 region with XMM-Newton (PI: Roberts) which was combined with archival observations for a total effective exposure (after removing times of high background) for each MOS detector of $\sim 105 \mathrm{ks}$. The complexity of the extended X-ray emission overlaid with infrared and radio emission is shown in Figure 8. A detailed analysis of these data will be presented elsewhere. Here we note that multi-color imaging (infrared, radio, and X-rays) shows that the extended emission centered on the pulsar is almost all non-thermal, and there is little evidence for strong spectral variation. The morphology of the X-ray emission to the west of the pulsar is that of a brighter tail of emission within a roughly bow-shaped nebula that conforms to the shape of the radio nebula. With the additional evidence of the same, approximate shape from the outer X-ray nebula to the west of the pulsar, we can plausibly assume that the cone-shaped region seen in the Very Large Array (VLA) image in Figure 8 starting at the pulsar and extending $10^{\prime}$ west belongs to the PWN of PSR J2021+3651.

If the shape is due to the pulsar's motion, then it is likely that the pulsar was born at least as far west as the apparent end of the radio tail. Given the pulsar's characteristic age of $\tau_{c} \sim 17000 \mathrm{yr}$, this implies a transverse motion of $\sim 0^{\prime \prime} 035 \mathrm{yr}^{-1}$. If we knew the distance to the pulsar, then we could estimate its transverse velocity. Unfortunately, the distance to PSR J2021+3651 is controversial. The dispersion measure is quite large, which yields an estimated distance of $\sim 12 \mathrm{kpc}$ from the NE 2001 free electron model (Cordes \& Lazio 2002). Roberts et al. (2002) pointed out that such a large distance would imply a very high $\gamma$-ray efficiency compared with other known pulsars, and a PWN X-ray efficiency at the high end of the distribution for young pulsars. Considering the X-ray emission and absorption, as well as excess scattering of the radio pulse indicating line of sight material not accounted for in the NE2001 model, Hessels et al. (2004) suggested a distance of $\sim 8 \mathrm{kpc}$ might be more reasonable. Other authors argued that, despite the large radio dispersion measure, the nebular size and the thermal $\mathrm{X}$-ray emission from the pulsar surface indicated a distance as low as $4 \mathrm{kpc}$ (Van Etten et al. 2008). However, the sense and 


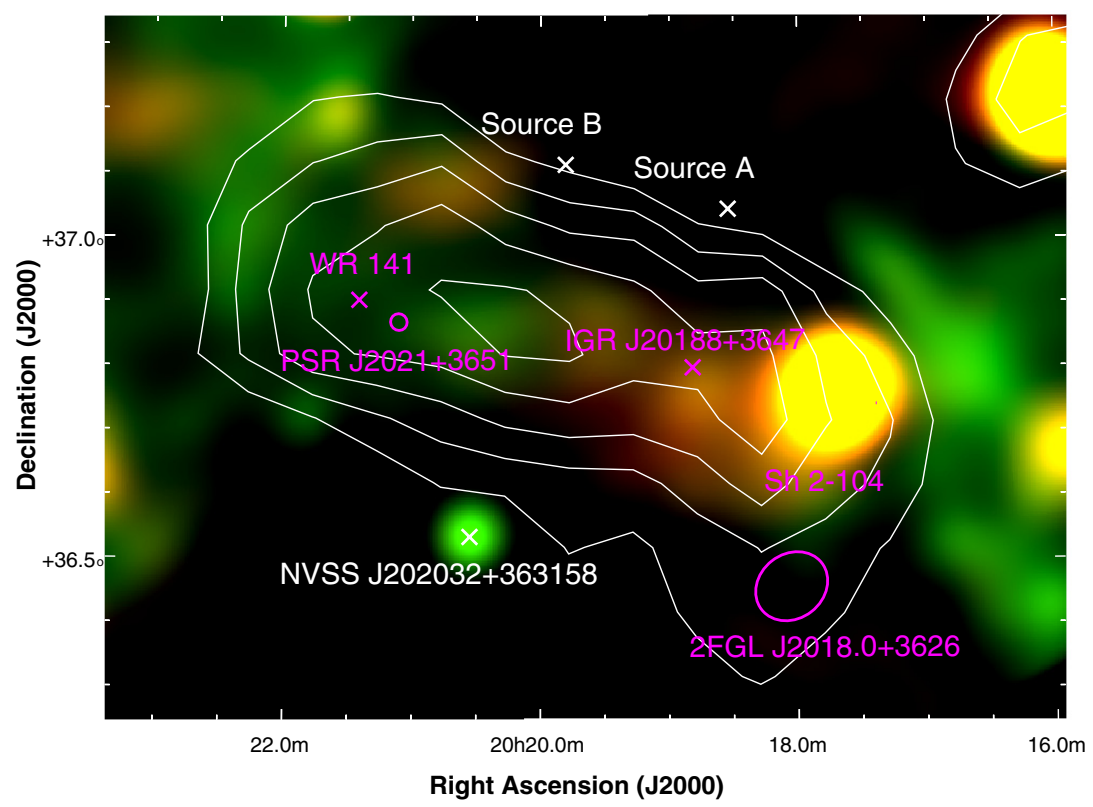

Figure 7. False color image of the radio diffuse emission of the VER J2019+368 region obtained with CGPS $408 \mathrm{MHz}$ (green) and GB6 $6 \mathrm{~cm}$ (red). To produce this image, we have taken the CGPS and GB6 radio images of the region and convolved them with the same beam size. The VERITAS significance contours at 3, 4, 5, 6 , and $7 \sigma$ obtained with the large integration radius $(0.23)$ are overlaid. Possible counterparts to MGRO J2019+37 within the literature are marked and labeled in magenta if they fall inside the $3 \sigma$ contours of VER J2019+368, and white otherwise.

(A color version of this figure is available in the online journal.)

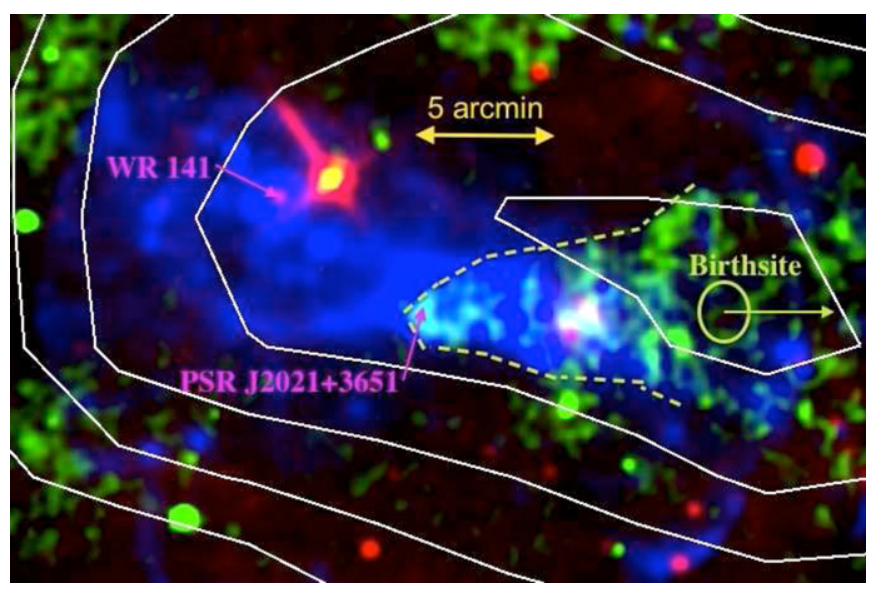

Figure 8. False color image of the infrared, radio, and X-ray emission in the vicinity of PSR J2021+3651 obtained with MSX $8.3 \mu \mathrm{m}$ (red), VLA $20 \mathrm{~cm}$ (green), and XMM 1-8 keV (blue), respectively. The VERITAS significance contours at $3,4,5,6$, and $7 \sigma$ obtained with the large integration radius $(0.23)$ are also shown. Overlaid dashed line highlights the PWN morphology in radio, and an ellipse marks the closest birthsite suggested by this morphology. An arrow indicates the direction of the birthsite if it happened further away.

(A color version of this figure is available in the online journal.)

magnitude of the polarization rotation measure seem to imply a minimum distance of $\sim 5 \mathrm{kpc}$ (Abdo et al. 2009b). Assuming a distance of $5 \mathrm{kpc}$, the implied transverse velocity of the pulsar is $v=840 d_{5} \tau_{17}^{-1} \mathrm{~km} \mathrm{~s}^{-1}$, where $\tau_{17}=\tau / 17000 \mathrm{yr}$ and $d_{5}=d / 5 \mathrm{kpc}$. This velocity is 3-4 times higher than the average for pulsar proper velocities, but would not be record breaking (Chatterjee et al. 2005). Since the true age is very unlikely to be greater than twice the characteristic age (Kaspi et al. 2001), the pulsar most likely could not have been born much farther west than the extent of the radio tail (Figure 8) unless its actual distance from Earth is much smaller than $\sim 5 \mathrm{kpc}$. In particular, it is unlikely that the pulsar was born near the west edge of the VHE emission, which is an additional $\sim 30^{\prime}$ further west and makes it less likely to be responsible for the entire VHE emission.

X-ray measurements of PSR J2021+3651 reveal that its properties are similar to those of the nearby young pulsar Vela$\mathrm{X}$. Both pulsars have high spin-down luminosities (the spindown luminosity of Vela-X is twice that of PSR J2021+3651) and possess compact inner toruses (Roberts et al. 2002; Van Etten et al. 2008). Also, the remarkably hard spectrum of VER J2019+368 is similar to the hard TeV emission that was observed in Vela-X (Abramowski et al. 2012). Although a single VHE PWN scenario may not be plausible to explain the entire VHE emission, based on the distance and velocity estimation, emission from the PWN is likely responsible for a significant portion of the emission.

\subsubsection{IGR J20188+3647}

The location of the hard X-ray transient IGR J20188+3647 lies near the maximum of the VHE emission. This transient was reported in 2004 July, and its flaring behavior consisted of a fast rise ( $\sim 10$ minutes) followed by a slower decay ( $\sim 50$ minutes) (Sguera 2008). Following a flare detected by AGILE in the region, a $\sim 21 \mathrm{ks} X M M$-Newton observation took place in 2007 November around IGR J20188+3647 (Zabalza et al. 2010). In that observation, five X-ray candidate sources appear inside the error box of the INTEGRAL source of 3.4. Four of them are associated with late stellar types, while the fifth one, which appears to be a highly absorbed hard X-ray source, is considered to be either an active galactic nucleus or a pulsar. We carried out observations with the Swift X-ray telescope (Swift-XRT) spread over three months (2011 June, July, and August 16th, PI: Aliu), aiming at finding some brightening of these $\mathrm{X}$-ray sources to identify a potential counterpart for this transient, but no likely counterparts were found. 


\subsubsection{Sh $2-104$}

The western edge of VER J2019+368 overlaps the H II region Sh 2-104. At a distance of $\sim 4 \mathrm{kpc}$, Sh 2-104 is beyond the Cygnus-X region. Sh 2-104 has a shell morphology in both optical and radio wavelengths with an O6V star in the center, which is ionizing the region. At the eastern edge of the shell, there is an ultracompact $\mathrm{HI}$ region, coincident with stellar clusters (Deharveng et al. 2003). With massive CO clouds around the star cluster, Deharveng et al. (2003) suggested that the Sh 2-104 region is a prototype of massive-star formation triggered by the expansion of an H II region. Star-forming regions and stellar clusters are considered to be possible gammaray sources, with the gamma-rays arising from shocks created by the wind of single or multiple massive stars colliding with the surrounding material (Torres et al. 2004). We cannot rule out the contribution from Sh 2-104 because of its nearby location to the $\mathrm{VHE}$ emission, although $\sim 6000 M_{\odot}$ of swept up mass of Sh 2-104 seems to be low compared to other star-forming regions that are associated with VHE gamma-ray sources, such as W49A (Brun et al. 2010) or Westerlund1 (Luna et al. 2010).

$$
\text { 4.2.5. } 2 F G L J 2018.0+3626
$$

An unassociated Fermi-LAT source, 2FGL J2018.0+3626, appears toward the southwest of VER J2019+368. This source is non-variable and shows a steep spectrum with a cutoff around $4 \mathrm{GeV}$, thus exhibiting properties similar to those of gammaray pulsars. Because the distance between 2FGL J2018.0+3626 and the centroid of VER J2019+368 is relatively large, it is unlikely that this source is directly responsible for the major part of the VHE emission. However, the softening at the west side of VER J2019+368 shown in Figure 4 could be associated with this secondary contribution. If the 2FGL source turns out to be an energetic pulsar, then it is possible for a PWN to contribute part of the west side of the VHE emission. To check this scenario, identifying the nature of 2FGL J2018.0+3626 is necessary. Unfortunately, the region is not well observed in $\mathrm{X}$-rays, making a search for a possible pulsar or a PWN difficult. The position of 2FGL J2018.0+3626 is outside the FOV of the XMM-Newton search reported by Zabalza et al. (2010). The low resolution radio surveys suggest some possible faint emission, but it is hard to quantify because of nearby bright emissions from Sh 2-104.

\subsubsection{Other Suggested Counterparts}

Paredes et al. (2009) searched the region for possible counterparts to the Milagro emission by using GMRT $610 \mathrm{MHz}$ data. Together with PSR J2021+3651 and the H II region Sh 2-104 that we mentioned above, Paredes et al. (2009) suggested the possibility of contributions from three non-thermal sources with jet-like structures. The improved VHE image of the region obtained with VERITAS excludes these three, labeled as sources A, B, and NVSS J202032+363158, which are marked in white in Figure 7. These sources fall outside the $3 \sigma$ emission region from VERITAS.

\section{SUMMARY AND CONCLUSION}

We have carried out a deep VHE observation in the region of MGRO J2019+37 with VERITAS, confirming this to be a very gamma-ray-rich area of the Galactic Plane with emission detected also with Fermi-LAT and Milagro, and thus covering the full range of the high energy spectrum.
The new VHE image spatially resolves, for the first time, one Milagro extended source into at least two clearly separate sources. The angular resolution is good enough to exclude some scenarios that were proposed for MGRO J2019+37. The most likely counterpart of the new source VER J2016+371 is the PWN in the SNR CTB 87. The co-location, the VHE extent, and the X-ray/VHE luminosity ratio argue in favor of this. More complicated is the multi-wavelength emission from VER J2019+368, as there are a few possible explanations for its physical origin. The young and energetic pulsar PSR J2021+3651 and its PWN, proposed by many before, seems a likely contributor to VER J2019+368, and therefore MGRO J2019+37. The extended VHE morphology in the direction of the X-ray and radio nebula favors this possibility. The very hard spectrum of the source, $\Gamma=1.75 \pm 0.08_{\text {stat }} \pm 0.3_{\text {sys }}$, which resembles that of Vela X (Aharonian et al. 2006), another PWN system, also favors this scenario. However, the picture might be more complex as non-thermal X-ray emission coincident with Sh 2-104 and an unassociated Fermi-LAT source are also in physical association with this large VHE emission. Follow-up multi-wavelength observations of the region, including VHE data, will certainly help clarify which scenario or scenarios are in fact originating VER J2019+368.

The authors would like to thank Jules Halpern for many useful discussions and help in obtaining and interpreting the Swift data. This research is supported by grants from the U.S. Department of Energy Office of Science, the U.S. National Science Foundation, and the Smithsonian Institution, by NSERC in Canada, by Science Foundation Ireland (SFI 10/RFP/AST2748), and by STFC in the U.K. We acknowledge the excellent work of the technical support staff at the Fred Lawrence Whipple Observatory and at the collaborating institutions in the construction and operation of the instrument. Based on observations obtained with XMM-Newton, an ESA science mission with instruments and contributions directly funded by ESA Member States and NASA. This research has made use of data and/or software provided by the High Energy Astrophysics Science Archive Research Center (HEASARC), which is a service of the Astrophysics Science Division at NASA/GSFC and the High Energy Astrophysics Division of the Smithsonian Astrophysical Observatory. The research presented in this paper has used data from the Canadian Galactic Plane Survey, a Canadian project with international partners, supported by the Natural Sciences and Engineering Research Council.

Facility: VERITAS

\section{REFERENCES}

Abdo, A. A., Abeysekara, U., Allen, B.T., et al. 2012, ApJ, 753, 159 Abdo, A. A., Ackermann, M., Ajello, M., et al. 2009b, ApJ, 700, 1059 Abdo, A. A., Allen, B. T., Aune, T., et al. 2009a, ApJ, 700, L127 Abdo, A. A., Allen, B. T., Berley, D., et al. 2007a, ApJ, 658, L33 Abdo, A. A., Allen, B. T., Berley, D., et al. 2007b, ApJ, 664, L91 Abramowski, A., Acero, F., Aharonian, F., et al. 2011, A\&A, 528, 143 Abramowski, A., Acero, F., Aharonian, F., et al. 2012, A\&A, 548, A38 Acciari, V., Beilicke, M., Blaylock, G., et al. 2008, ApJ, 679, 1427 Aharonian, F., Akhperjanian, A. G., Aye, K.-M., et al. 2005, A\&A, 430, 865 Aharonian, F., Akhperjanian, A. G., Barrio, J., et al. 2001, A\&A, 370, 112 Aharonian, F., Akhperjanian, A. G., Bazer-Bachi, A. R., et al. 2006, A\&A, 448, 43

Aharonian, F. A., \& Atoyan, A. M. 1995, APh, 3, 275

Aliu, E., Archambault, S., Arlen, T., et al. 2013, ApJ, 764, 38 Amenomori, M., Bi, X. J., Chen, D., et al. 2008, ICRC, 2, 695 Bartko, H., Bednarek, W., Rico, J., et al. 2008, ICRC, 2, 649 Bartoli, B., Bernardini, P., Bi, X. J., et al. 2012, ApJ, 745, L22 
Bednarek, W. 2007, MNRAS, 382, 367

Brun, F., de Naurois, M., Hofmann, W., et al. 2010, in Proc. 25th Texas Symp. on Relativistic Astrophysics, ed. F. M. Rieger, C. van Eldik, \& W. Hofmann (Proceedings of Science), 201

Chatterjee, S., Vlemmings, W. H. T., Brisken, W. F., et al. 2005, ApJL, 630, L61

Cogan, P. 2008, ICRC, 3, 1385

Cordes, J. M., \& Lazio, T. J. W. 2002, arXiv:astro-ph/0207156v3

Deharveng, L., Lefloch, B., Zavagno, A., et al. 2003, A\&A, 408, L25

Gaensler, B. M., van derSwaluw, E., Camilo, F., et al. 2004, ApJ, 616, 383

Ginzburg, V. L., \& Syrovatskii, S. I. 1964, The Origin of Cosmic Rays (New York: Macmillan)

Hessels, J. W. T., Roberts, M. S. E., Ransom, S. M., et al. 2004, ApJ, 612, 389

Hillas, A. 1985, ICRC, 3, 445

Holder, J., Acciari, V. A., Aliu, E., et al. 2008, in AIP Conf. Proc. 1085, High Energy Gamma-Ray Astronomy, ed. F. A. Aharonian, W. Hoffmann, \& F. Rieger (Melville, NY: AIP), 657

Holder, J., Atkins, R. W., Badran, H. M., et al. 2006, APh, 25, 391

Kaaret, P., \& Cottam, J. 1996, ApJL, 462, L35

Kara, E., Errando, M., Max-Moerbeck, W., et al. 2012, ApJ, 746, 159

Kargaltsev, O., \& Pavlov, G. G. 2010, in Proc. X-Ray Astronomy 2009, ed. A. Comastri, M. Cappi, \& L. Angelini (Bologna, Italy: AIP)

Kargaltsev, O., Rangelov, B., \& Pavlov, G. G. 2013, in The Universe Evolution. Astrophysical and Nuclear Aspects, ed. Strakovsky \& Blokhintsev (Nova Science Publishers, Inc), arXiv:1305.2552

Kaspi, V. M., Roberts, M. E., Vasisht, G., et al. 2001, ApJ, 560, 371

Kieda, D. B. 2008, ICRC, 2, 843

Kothes, R., Reich, W., Foster, T., et al. 2003, ApJ, 588, 852

Lemoine-Goumard, M., Ferrara, E., Grondin, M.-H., et al. 2011, MmSAI, 82,739
Luna, A., Mayya, Y.D., Carrasco, L., et al. 2010, ApJL, 713, L45

Marchenko, S. V., Moffat, A. F. J., \& Eenens, P. R. J. 1998, PASP, 110,1416

Matheson, H., Safi-Harb, S., \& Kothes, R. 2013, ApJ, 774, 33

Montes, G., Pérez-Torres, M. A., Alberdi, A., \& González, R. F. 2009, ApJ, 705, 899

Montmerle, T. 1979, ApJ, 231, 95

Paredes, J. M., Martí, J., Ishwara-Chandra, C. H., et al. 2009, A\&A, 507, 241

Reimer, A., Pohl, M., \& Reimer, O. 2006, ApJ, 644, 1118

Roberts, M. S. E., Brogan, C., Ransom, S., et al. 2008, in AIP Conf. Proc. 1085 High Energy Gamma-Ray Astronomy, ed. F. A. Aharonian, W. Hoffmann, \& F. Rieger (Melville, NY: AIP), 328

Roberts, M. S. E., Hessels, J. W. T., Ransom, S. M., et al. 2002, ApJL, 577, L19

Romani, R. W., Ng, C.-Y., Dodson, R., \& Brisken, W. 2005, ApJ, 631, 480

Sguera, V. 2008, Proc. 7th INTEGRAL Workshop (Proceedings of Science), 82

Taylor, A. R., Gibson, S. J., Peracaula, M., et al. 2003, AJ, 125, 3145

Torres, D. F., Domingo-Santamaria, E., \& Romero, G. 2004, ApJL, 601, L75

Van der Hucht, K. A. 2001, NewAR, 45, 135

Van Etten, A., Romani, R. W., \& Ng, C.-Y. 2008, ApJ, 680, 1417

Wallace, B. J., Landecker, T. L., Taylor, A. R., \& Pineault, S. 1997, A\&A, 317 212

Weiler, K. W., \& Shaver, P. A. 1978, A\&A, 65, 305

Weinstein, A., \& for the VERITAS Collaboration. 2009, arXiv:0912.4492

Zabalza, V., Paredes, J. M., Martí, J., Sánchez-Sutil, J. R., \& Muñoz-Arjonilla, A. J. 2010, in ASP Conf. Ser. 422, High Energy Phenomena in Massive Stars, ed. J. Martí, P. L. Luque-Escamilla, \& J. A. Combi (San Francisco, CA: ASP), 186

Zhekov, S. A. 2012, MNRAS, 422, 1332 